Revista Destaques Acadêmicos, Lajeado, v. 12, n. 3, 2020. ISSN 2176-3070

DOI: http://dx.doi.org/10.22410/issn.2176-3070.v12i3a2020.2668

http://www.univates.br/revistas

\title{
QUALIDADE MICROBIOLÓGICA DE QUEIJOS ANALISADOS EM UM LABORATÓRIO DO RIO GRANDE DO SUL
}

\author{
Luana Elisa Forneck ${ }^{1}$, Fernanda Scherer Adami
}

Resumo: O objetivo do presente estudo foi analisar a associação entre o tipo de queijo, definido pela umidade, com o resultado conforme e não conforme para contaminação por Salmonella spp., Staphylococcus coagulase positiva e coliformes termotolerantes. Selecionaram-se 4.039 amostras de queijos, totalizando 11.163 resultados, do período de 2010 à 2017, realizados por um laboratório prestador de serviços analíticos. Os queijos foram classificados de acordo com a umidade e os resultados separados em conformes e não conformes, de acordo com o limite de tolerância aceitável de contaminação, valores estabelecidos pela legislação vigente. As metodologias utilizadas pelo laboratório para a realização das análises são AFNOR Validation 3M 01/02-09/89C para a análise de coliformes termotolerantes, ISO 6888-3 para Staphylococcus coagulase positiva e AOAC 2011.03 para Salmonella spp.. Os dados foram analisados pelos testes estatísticos de Associação Qui-quadrado e Teste Exato de Fisher. Foram identificadas como não conformes 8,95\% (346) de um total de 3865 análises para coliformes termotolerantes, 1,59\% (56) de 3526 análises de Staphylococcus coagulase positiva e 0,08\% (3) de 3772 análises para Salmonella spp.. Os queijos de muito alta e média umidade foram significativamente associados ao resultado de não conformidade e os queijos de alta e baixa umidade com o resultado de conformidade para coliformes termotolerantes $(\mathrm{p} \leq 0,01)$. Para Staphylococcus coagulase positiva foram significativamente associados os queijos de alta umidade ao resultado de conformidade e os queijos de média umidade ao resultado de não conformidade $(\mathrm{p}=0,001)$. Concluiu-se que os queijos de muito alta e média umidade foram significativamente associados ao resultado de não conformidade e os queijos de alta e baixa umidade com o resultado de conformidade em relação aos resultados sobre coliformes termotolerantes. Para Staphylococcus coagulase positiva, os queijos de alta umidade foram significativamente associados ao resultado de conformidade e os de média umidade ao resultado de não conformidade.

Palavras-chave: Queijo. Análise microbiológica. Qualidades dos alimentos.

1 Especialista em Gestão de Segurança Alimentar e Nutricional pela Universidade do Vale do Taquari - Univates, Lajeado/RS. Atua como laboratorista no Laboratório Unianálises Univates, Lajeado/RS.

2 Doutora em Ciências: Ambiente e Desenvolvimento - Univates, Lajeado/RS. Professora Titular da Universidade do Vale do Taquari - Univates, Lajeado/RS. 


\section{INTRODUÇÃO}

As Doenças Transmitidas por Alimentos (DTA), são uma preocupação frequente da cadeia alimentícia mundial. Com o advento da industrialização dos alimentos e o consumo global das mercadorias é de extrema necessidade que esse produto chegue à mesa do consumidor sem apresentar risco a saúde (TONDO; BARTZ, 2011). Segundo Mello e Armachuk (2013) fatores como a correta higienização e sanitização do ambiente de trabalho e dos equipamentos utilizados, a qualidade da matéria-prima e a manipulação durante todo o processo afetam a qualidade do produto. Controlar esses fatores é indispensável para que o risco presente no alimento seja reduzido ao máximo durante a sua produção, garantindo um risco aceitável, já que risco igual a zero se torna impraticável, devido à alta gama de produtos disponíveis no mercado (FORSYTHE, 2013). Bradley Jr., Houck e Smukowski (2015) afirmam que no caso de serem encontrados microrganismos patógenos, há um grande indício de falhas no processo de produção e controle de matrizes.

Dentre os surtos epidemiológicos registrados no Brasil, as bactérias representam em torno de $90 \%$ dos casos. Os coliformes termotolerantes, Salmonella spp. e Staphylococcus coagulase positiva são considerados os três maiores causadores de surtos de doenças transmitidas por alimentos (BRASIL, 2016).

Os coliformes termotolerantes são microrganismos diretamente relacionados à higiene inadequada no processamento dos alimentos (MADIGAN; MARTINKO; PARKER, 2004). Da mesma forma, a presença de Salmonella spp. é um indício de contato fecal, também demonstrando deficiência na higienização durante o processo de produção (TORTORA; FUNKE; CASE, 2012). Por apresentar um risco de infecção maior para o organismo que se contamina com ela, a legislação brasileira determina a ausência desse microrganismo no produto (BRASIL, 1996).

Outro microrganismo que necessita de atenção é o Staphylococcus coagulase positiva que está diretamente ligado a sanidade do gado leiteiro, sendo o principal microrganismo causador de mastite. Caso o leite derivado de um animal doente seja pasteurizado de maneira insatisfatória e contenha um número elevado de microrganismos, o risco de uma enterotoxicose estafilocócica é alto. O Staphylococcus coagulase positiva é extremamente importante no controle de processos de alimentos, pois sua presença detectada após o processo, também pode indicar sanitização inadequada de manipuladores e/ ou superfícies (BENNETT; HAIT; TALLENT, 2016).

$\mathrm{O}$ queijo é uma rica fonte de nutrientes o que acaba favorecendo a proliferação de microrganismos e pode levar a toxinfecções alimentares nos consumidores (MARINHEIRO et al., 2015). Porém, é importante ressaltar que outros microrganismos são desejáveis, e tem um papel primordial na produção 
e fermentação do queijo, pois garantem que o produto apresente características organolépticas próprias para cada tipo de queijo (MELLO; ARMACHUK, 2013).

Garantir que não haja contaminação cruzada ou que a população presente de microrganismos não se prolifere consideravelmente, a ponto de causar malefícios aos consumidores, é uma obrigação da indústria produtora e do Estado. Para garantir que o produto final comercializado seja considerado seguro ao consumo foram criadas legislações, como a Portaria $\mathrm{n}^{\circ} 146$, de 7 de março de 1996, que determina os padrões microbiológicos que garantem a sanidade do processo de fabricação dos queijos (BRASIL, 1996).

O Regulamento Técnico de Identidade e Qualidade de Queijos, Portaria $\mathrm{N}^{\mathrm{o}}$ 146, de 7 de março de 1996, fixa a identidade dos queijos definindo que:

Entende-se por queijo o produto fresco ou maturado que se obtém por separação parcial do soro do leite ou leite reconstituído (integral, parcial ou totalmente desnatado), ou de soros lácteos, coagulados pela ação física do coalho, de enzimas específicas, de bactérias específicas, de ácidos orgânicos, isolados ou combinados, todos de qualidade apta para uso alimentar, com ou sem agregação de substâncias alimentícias e/ou especiarias e/ou condimentos, aditivos especificamente indicados, substâncias aromatizantes e matérias corantes (BRASIL, 1996).

O objetivo do presente estudo foi analisar a associação entre o tipo de queijo, definido pela umidade, com o resultado conforme e não conforme para contaminação por coliformes termotolerantes, Staphylococcus coagulase positiva e Salmonella spp., com a finalidade de avaliar o padrão de controle de qualidade das empresas e da fiscalização sanitária.

\section{MATERIAIS E MÉTODOS}

Realizou-se uma pesquisa quantitativa retrospectiva em um software utilizado para expressar resultados de análises microbiológicas de um laboratório prestador de serviços analíticos do Rio Grande do Sul com certificações MAPA, Inmetro, CISPOA, Fepam.

Selecionaram-se 4.039 amostras de queijo, sendo os seus resultados analisados para coliformes termotolerantes e/ou Salmonella spp. e/ou Staphylococcus coagulase positiva, de janeiro de 2010 até abril de 2017. Os dados selecionados totalizaram 11.163 resultados, sendo $3865(34,62 \%)$ para a análise de coliformes termotolerantes, $3526(31,59 \%)$ para Staphylococcus coagulase positiva e 3772 (33,79\%) para Salmonella spp.

As metodologias utilizadas pelo laboratório para a realização das análises são AFNOR Validation 3M 01/02-09/89C para a análise de coliformes termotolerantes, ISO 6888-3 para Staphylococcus coagulase positiva e AOAC 2011.03 para Salmonella spp. (BRASIL, 2017). 
Os queijos selecionados foram separados de acordo com a umidade estabelecida pela portaria $\mathrm{n}^{\circ} 146$, que classifica os queijos conforme a porcentagem de umidade, sendo considerados de baixa umidade até $35,9 \%$ (queijos de massa dura), média umidade entre 36,0\% e 45,9\% (queijos de massa semidura), de alta umidade entre $46,0 \%$ e $54,9 \%$ (massa branda ou "macios") e muito alta umidade não inferior a 55,0\% (queijos de massa branda ou "mole") (BRASIL, 1996). Cada tipo de queijo possui uma portaria específica que determina seu regulamento técnico e fixa sua identidade e qualidade, garantindo a padronização do processo de elaboração do produto. Essas portarias foram utilizadas para a classificação dos queijos de acordo com a umidade, conforme Quadro 1.

Quadro 1 - Classificação dos queijos de acordo com a umidade.

\begin{tabular}{|c|c|c|}
\hline $\begin{array}{l}\text { Umidade do } \\
\text { queijo }\end{array}$ & Variedade do queijo & Legislação \\
\hline \multirow[t]{2}{*}{ Baixa umidade } & $\begin{array}{l}\text { Parmesão, } \\
\text { Parmesano, } \\
\text { Sbritz, Reggiano e } \\
\text { Reggianito }\end{array}$ & Portaria $\mathrm{n}^{\circ} 353$ (BRASIL, 1997) \\
\hline & Queijo em pó & Portaria n 355 (BRASIL, 1997) \\
\hline \multirow{10}{*}{ Média umidade } & $\begin{array}{l}\text { Prato, Lanche, } \\
\text { Sandwich, Cobocó }\end{array}$ & Portaria nº 358 (BRASIL, 1997) \\
\hline & Azul & Instrução Normativa n ${ }^{\circ} 45$ (BRASIL, 2007) \\
\hline & Danbo & Portaria n ${ }^{\circ} 360$ (BRASIL, 1997) \\
\hline & Ralado & Portaria no 357 (BRASIL, 1996) \\
\hline & Tilsit & Portaria nº 361 (BRASIL, 1997) \\
\hline & Tropical & Instrução Normativa n $^{\circ} 24$ (BRASIL, 2002) \\
\hline & Coalho & $\begin{array}{l}\text { Instrução Normativa n }{ }^{\circ} \text { 30, Anexo II } \\
\text { (BRASIL, 2001) }\end{array}$ \\
\hline & Tybo & Portaria nº 362 (BRASIL, 1997) \\
\hline & Pategrás Sandwich & Portaria nº 363 (BRASIL, 1997) \\
\hline & Tandil & Portaria $n^{\circ} 365$ (BRASIL, 1997) \\
\hline Alta umidade & Mussarela & Portaria n 364 (BRASIL, 1997) \\
\hline
\end{tabular}




\begin{tabular}{|l|l|l|}
\hline $\begin{array}{l}\text { Umidade do } \\
\text { queijo }\end{array}$ & Variedade do queijo & Legislação \\
\hline \multirow{2}{*}{$\begin{array}{l}\text { Muito alta } \\
\text { umidade }\end{array}$} & Minas frescal & $\begin{array}{l}\text { Portaria } n^{\circ} 352 \text { (BRASIL, 1997) / Instrução } \\
\text { Normativa } n^{\circ} 4 \text { (BRASIL, 2004) }\end{array}$ \\
\cline { 2 - 3 } & Requeijão & Portaria $n^{\circ} 359$ (BRASIL, 1997) \\
\cline { 2 - 3 } & Petit Suisse & Instrução Normativa n ${ }^{\circ} 53$ (BRASIL, 2000). \\
\hline
\end{tabular}

Fonte: Da autora.

Quando a legislação específica do produto permitia mais de uma possibilidade de umidade, para determinado tipo de queijo, foi selecionada a que apresentava o menor limite de contaminação aceitável. As amostras que não apresentavam legislação para o tipo de queijo foram desconsideradas.

Após a classificação quanto a umidade, as amostras foram separadas conforme o limite estabelecido pela legislação vigente, sendo divididas em amostras conformes e não conformes. Dentro do regulamento técnico específico de cada tipo de queijo encontram-se os critérios microbiológicos que determinam os limites de tolerância aceitáveis de contaminação, vezes orientado na própria legislação, vezes determinada pela portaria n¹46 (BRASIL, 1996).

Os dados foram analisados através de tabelas, estatísticas descritivas e pelos testes estatísticos de Associação Qui-quadrado e Teste Exato de Fisher. Os resultados foram considerados significativos a um nível de significância máximo de $5 \%(p \leq 0,05)$ e o software utilizado para esta análise foi o SPSS versão 22.0.

\section{RESULTADOS}

O estudo avaliou 11.163 análises, sendo de 4.039 amostras de queijos com teor de umidade diferentes, demonstrando resultados não conformes para 8,95\% (346) de um total de 3865 análises para coliformes termotolerantes, 1,59\% (56) de 3526 análises para Staphylococcus coagulase positiva e 0,08\% (3) de 3772 análises para Salmonella spp..

Na Tabela 1 verificou-se associação significativa do resultado para coliformes termotolerantes com o tipo de queijo. Os queijos de muito alta e média umidade foram significativamente associados ao resultado de não conformidade e os queijos de alta e baixa umidade com o resultado de conformidade para coliformes termotolerantes $(\mathrm{p} \leq 0,01)$. 
Tabela 1 - Associação entre o tipo de queijo e a contaminação por coliformes termotolerantes.

\begin{tabular}{|c|c|c|c|c|c|}
\hline \multirow[b]{3}{*}{ Tipo de Queijo } & \multicolumn{4}{|c|}{ Coliformes termotolerantes } & \multirow[b]{3}{*}{$\mathrm{p}$} \\
\hline & \multicolumn{2}{|c|}{ Conforme } & \multicolumn{2}{|c|}{ Não conforme } & \\
\hline & $\mathrm{n}$ & $\%$ & $\mathrm{n}$ & $\%$ & \\
\hline Muito alta umidade & 218 & $6,2 \%$ & 57 & $16,5 \%$ & $\mathrm{p} \leq 0,01$ \\
\hline Alta & 1569 & $44,6 \%$ & 34 & $9,8 \%$ & \\
\hline Média & 1410 & $40,1 \%$ & 247 & $71,4 \%$ & \\
\hline Baixa & 322 & $9,2 \%$ & 8 & $2,3 \%$ & \\
\hline Total & 3519 & $100,0 \%$ & 346 & $100,0 \%$ & \\
\hline
\end{tabular}

Teste Qui-quadrado.

$\mathrm{Na}$ Tabela 2 os queijos de alta umidade foram significativamente associados, $(p=0,001)$, ao resultado de conformidade e os queijos de média umidade ao resultado de não conformidade em relação ao Staphylococcus coagulase positiva e o tipo de queijo.

Tabela 2 - Associação entre o tipo de queijo e a contaminação por Staphylococcus coagulase positiva.

\begin{tabular}{l|c|c|c|c|c}
\hline \multirow{2}{*}{ Tipo de Queijo } & \multicolumn{4}{|c|}{ Staphylococcus coagulase positiva } & \multirow{2}{*}{} \\
\cline { 2 - 5 } & \multicolumn{2}{|c|}{ Conforme } & \multicolumn{2}{c}{ Não conforme } & \multirow{2}{*}{$\mathrm{p}$} \\
\cline { 2 - 5 } Muito alta umidade & $\mathrm{n}$ & $\mathrm{\%}$ & $\mathrm{n}$ & $\%$ & 0,001 \\
\hline Alta & 418 & $12,0 \%$ & 5 & $8,9 \%$ & \\
\hline Média & $\mathbf{1 4 4 6}$ & $\mathbf{4 1 , 7} \%$ & 10 & $17,9 \%$ & \\
\hline Baixa & 1414 & $40,7 \%$ & $\mathbf{3 5}$ & $\mathbf{6 2 , 5 \%}$ & \\
\hline Total & 192 & $5,5 \%$ & 6 & $10,7 \%$ & \\
\hline
\end{tabular}

Teste Qui-quadrado.

A Tabela 3 demostrou que não houve associação significativa entre os diferentes tipos de queijo para a conformidade e não conformidade para Salmonella spp. $(\mathrm{p}=0,518)$. 
Tabela 3 - Associação entre o tipo de queijo e a contaminação por Salmonella spp..

\begin{tabular}{l|c|c|c|c|c}
\hline \multirow{2}{*}{ Tipo de Queijo } & \multicolumn{4}{|c|}{ Salmonella spp. } & \multirow{2}{*}{} \\
\cline { 2 - 5 } & \multicolumn{2}{|c|}{ Conforme } & \multicolumn{2}{c}{ Não conforme } & \multirow{2}{*}{$\mathrm{p}$} \\
\cline { 2 - 5 } & $\mathrm{n}$ & $\%$ & $\mathrm{n}$ & $\%$ & 0,518 \\
\hline Muito alta umidade & 363 & $9,6 \%$ & - & - & \\
\hline Alta & 1560 & $41,4 \%$ & 1 & $33,3 \%$ & \\
\hline Média & 1491 & $39,6 \%$ & 1 & $33,3 \%$ & \\
\hline Baixa & 355 & $9,4 \%$ & 1 & $33,3 \%$ & \\
\hline Total & 3769 & $100,0 \%$ & 3 & $100,0 \%$ & \\
\hline
\end{tabular}

Teste Exato de Fisher.

\section{DISCUSSÃO}

Ao avaliar a carga microbiana de coliformes termotolerantes, EvangelistaBarreto et al. (2016) encontraram 70\% (10) das amostras de queijo coalho acima dos parâmetros estabelecidos pela legislação, assim como Adami et al. (2015) que apresentaram 41,7\% (10) para queijos prato e colonial, Marinheiro et al. (2015) que avaliaram queijos Mussarela e relataram 12,5\% (5) e Oliveira et al. (2017) que obtiveram 27,8\% (10) das amostras de queijo Minas frescal acima do limite, ambos resultados superiores aos observados no presente estudo. Já em um estudo realizado no Sudoeste do Paraná com amostras de laticínios inspecionadas pelo Serviço de Inspeção de Origem Animal (SIP/POA) nenhuma amostra de queijo mussarela foi considerada em desacordo para coliformes termotolerantes, a correta educação e assistência dos produtores de queijo, em todas as etapas, é um fator determinante para que o produto final seja de qualidade (RAIMANN, 2011).

Para a análise de Staphylococcus coagulase positiva, no presente estudo, demonstrou-se que 1,59\% (56) das amostras estão não conformes para os parâmetros legais de todos os tipos de queijo analisados, enquanto que para Marinheiro et al. (2015) foram detectadas 10\% (4) do total de amostras de queijo mussarela em desacordo, Adami et al. (2015) encontraram em suas análises $25 \%$ (5) em queijos prato e colonial em não conformidade e Evangelista-Barreto et al. (2016), 86\% (12) quando avaliaram o queijo coalho produzido com leite cru. Também houve um estudo que não detectou a presença do microrganismo em queijo Mussarela, analisado no estado do Paraná (RAIMANN, 2011). No estudo realizado por Rezende et al. (2010), com queijos Minas artesanal, todas as amostras apresentaram resultados acima dos padrões estabelecidos para Staphylococcus coagulase positiva. Da mesma forma que para os resultados de coliformes termotolerantes, os autores (REZENDE et al., 2010) atribuem 
esse valor as condições higiênico-sanitárias inadequadas na produção e comercialização do produto analisado. Mesmo em queijos de diferentes níveis de umidade a questão higiênica sanitária é de fundamental importância para garantir um alimento seguro, o que é indispensável para a proteção da saúde pública (SILVA et al., 2017).

Marinheiro et al. (2015) encontraram 2,5\% (1) das amostras com a presença de Salmonella spp., resultado superior ao encontrado no presente estudo. Já em outros estudos não foi detectada a presença deste microrganismo (ADAMI et al., 2015; EVAGELISTA-BARRETO et al., 2016; GALINARI et al., 2014; RAIMANN, 2011; REZENDE et al. 2010). A prevalência de resultados negativos ou mesmo com poucos casos de contaminação pode ser devido a Salmonella spp. possuir uma discreta incidência no leite, podendo ocorrer quando o rebanho apresenta a doença, quando o manipulador, no momento da produção, é portador, no caso de ser utilizada água não potável para a produção ou ainda pelo fato do microrganismo não ser um bom competidor, já que sofre injúria em produtos que apresentam alta carga de contaminação por coliformes (TORTORA; FUNKE; CASE, 2012).

Para a análise de coliformes termotolerantes, os queijos de muito alta e média umidade foram significativamente associados ao resultado de não conformidade e os queijos de alta e baixa umidade com o resultado de conformidade. $\mathrm{O}$ fato dos resultados significativos terem ficado intercalados, entre conformes e não conformes, com relação à umidade, demostra que pode haver outro fator influenciando na multiplicação de microrganismos, como as condições sanitárias do rebanho, a qualidade do leite, as distintas condições de fabricação do queijo, transporte, comercialização, tempo e temperatura de conservação, durante a estocagem, podem resultar em diferentes níveis de contaminação, que são primordiais para a qualidade do produto final (REZENDE et al., 2010).

Estudos realizados com queijos consideraram seus resultados como insatisfatórios para o consumo humano, devido à contaminação por microrganismos patogênicos, reflexo da falta de cuidados na higienização no momento da produção, na qualidade da matéria-prima selecionada, no transporte e armazenamento inadequado (EVAGELISTA-BARRETO et al., 2016; SILVA; FURTADO; VARGAS, 2017; OLIVEIRA et al., 2017; SILVA et al., 2017). Confirmando que a adequação às Boas Práticas de Fabricação é um fator importante para determinar a qualidade do produto final (MARINHEIRO et al. 2015).

Uma limitação do estudo pode ter ocorrido devido ao fato de não ter sido quantificada a umidade, através de análise, e sim, separada em classes de umidades através dos padrões referenciados pelas legislações para realizar a relação entre os dois parâmetros. São necessários mais estudos que avaliem a relação da umidade do queijo com a carga microbiana para identificar a relevância do fator umidade na produção dos diferentes tipos de queijo. 


\section{CONCLUSÃO}

Os queijos de muito alta e média umidade foram significativamente associados ao resultado de não conformidade e os queijos de alta e baixa umidade com o resultado de conformidade em relação aos resultados sobre coliformes termotolerantes. Em relação aos resultados sobre Staphylococcus coagulase positiva, os queijos de alta umidade foram significativamente associados ao resultado de conformidade e os queijos de média umidade ao resultado de não conformidade. Já em relação aos resultados da análise para Salmonella spp não verificou-se associação significativa entre a conformidade e não conformidade dos diferentes tipos de queijo.

Os resultados do presente estudo apontam baixos índices de contaminação por coliformes termotolerantes, Staphylococcus coagulase positiva e Salmonella spp. nas amostras analisadas, o que demonstra que o controle de qualidade na produção dos queijos e o sistema de fiscalização vem sendo efetivos. Entretanto, ainda é evidenciado que algumas amostras estão sendo produzidas fora dos parâmetros estabelecidos pela legislação para contaminação por microrganismos patógenos e, se esse produto for consumido antes da sua detecção, pode vir a causar risco à saúde do consumidor. Isso demonstra a importância da atuação incisiva dos órgãos de fiscalização sanitária, bem como o interesse e cuidado dos fabricantes durante o processo e antes de liberar o produto final para a comercialização.

\section{REFERÊNCIAS}

ADAMI, Fernanda S.; DAL BOSCO, Simone M.; ALTENHOFEN, Gabriela; SOUZA, Claucia F. V. de; OLIVEIRA, Eniz C. Avaliação da qualidade microbiológica de linguiças e queijos. Caderno pedagógico, Lajeado, v. 12, n. 1, p. 46-55, 2015. ISSN 1983-0882. Disponível em: < http:// univates.br/revistas/ index.php/cadped/article/ view/931/919>. Acesso em: 7 abr. 2017.

BENNETT, Reginald W.; HAIT, Jennifer M.; TALLENT, Sandra M. Staphylococcus aureus and Staphylococcal Enterotoxins. In: SALFINGER, Yvonne; TORTORELLO, Mary L. (Ed.). Compendium of Methods: for the Microbiological Examination of Foods. 5. ed. Washington, DC: APHA Press, 2016. p. 509-511.

BRADLEY JR, Robert L.; HOUCK, Kristin; SMUKOWSKI, Marianne. Milk and milk products. In: SALFINGER, Yvonne; TORTORELLO, Mary L. (Ed.). Compendium of Methods: for the Microbiological Examination of Foods. 5. ed. Washington, DC: APHA Press, 2015. p.645-653

BRASIL. Ministério da Agricultura Pecuária e Abastecimento. Secretaria Nacional de Defesa Agropecuária. DOC SAC/CGAL no 04: Escopo da Área de Microbiologia em Alimentos e Águas - Revisão 12. Disponível em: < http:/ / www.agricultura.gov.br/assuntos/laboratorios/ laboratorios-credenciados/ 
documentos-rede-nacional-de-laboratorios agropecuarios/DOC04POA

microbiolgicosALIMENTOS01_02_2018Reviso12.pdf>. Acesso em: 5 fev. 2018.

BRASIL. Portaria n. 146, de 07 de março de 1996. Aprova os regulamentos técnicos de identidade e qualidade dos produtos lácteos. Disponível em: < http:/ / extranet.agricultura.gov.br/sislegis-consulta/consultar Legislacao.do? operacao=visualizar\&id=1218>. Acesso em: 20 abr. 2017.

BRASIL. Ministério da Saúde. Secretaria de Vigilância em Saúde. Surtos de Doenças Transmitidas por Alimentos no Brasil. 2016. Disponível em: $<$ http:/ / portalarquivos. saude.gov.br/images/pdf/2016/junho/08/Apresenta----o-Surtos-DTA-2016.pdf>. Acesso em: 6 jun. 2017.

BRASIL. Ministério da Agricultura, Pecuária e Abastecimento. Instrução Normativa $\mathrm{n}^{\mathrm{o}}$ 4, de 1 de março de 2004. Brasília, DF. Disponível em: <http:/ / extranet.agricultura. gov.br/sislegis- consulta/consultarLegislacao.do?operacao=visualizar\&id=5974>. Acesso em: 06 jun. 2017.

BRASIL. Ministério da Agricultura, Pecuária e Abastecimento. Instrução Normativa $\mathrm{n}^{\circ} 24$, de 4 de abril de 2002. Regulamento técnico para fixação de identidade e qualidade do queijo regional do Norte ou queijo Tropical de uso industrial. Brasília, DF. Disponível em: <http:/ / wp.ufpel.edu.br/inspleite/files/2016/03/ Instru \%C3\%A7\%C3\%A3o-normativa-n \%C2\%B0-24-de-4-de-abril-de-2002.pdf $>$. Acesso em: 06 jun. 2017.

BRASIL. Ministério da Agricultura, Pecuária e Abastecimento. Instrução Normativa $\mathrm{n}^{\circ} 30$ de 2 de junho de 2001. Regulamento técnico para fixação de identidade e qualidade de Manteiga da Terra ou Manteiga de Garrafa; Queijo de Coalho e Queijo de Manteiga. Brasília, DF. Disponível em: < https:/ /wp.ufpel.edu.br/ inspleite/files / 2016/03/Instru\%C3\%A7\%C3\%A3o-normativa-n \%C2\%B0-30-de-26-dejunho-de-2001.pdf $>$.

BRASIL. Ministério da Agricultura, Pecuária e Abastecimento. Instrução Normativa $\mathrm{n}^{\circ} 45$, de 23 de outobro de 2007. Regulamento técnico para fixação de identidade e qualidade do queijo Azul. Brasília, DF. Disponível em: <http:/ / wp.ufpel.edu.br/ inspleite/files / 2016/03/Instru\% C3\%A7\%C3\%A3o-normativa-n\%C2\%B0-45-de-23de-outubro-de-2007.pdf>. Acesso em: 06 jun. 2017.

BRASIL. Ministério da Agricultura, Pecuária e Abastecimento. Instrução Normativa $\mathrm{n}^{\circ}$ 53, de 29 de dezembro de 2000. Regulamento técnico para fixação de identidade e qualidade do queijo "Petit Suisse". Brasília, DF. Disponível em: <http:/ / extranet. agricultura.gov.br/sislegis- consulta/servlet/VisualizarAnexo?id=1605>. Acesso em: 06 jun. 2017.

BRASIL. Ministério da Agricultura, Pecuária e Abastecimento. Portaria no 352 de 04 de setembro de 1997. Regulamento técnico para fixação de identidade e qualidade do queijo Minas Frescal. Brasilia, DF. Disponível em: <http:/ / extranet.agricultura. gov.br/sislegis-consulta/ servlet/VisualizarAnexo?id=12874>. Acesso em: 06 jun. 2017. 
BRASIL. Ministério da Agricultura, Pecuária e Abastecimento. Portaria no 353 de 04 de setembro de 1997. Regulamento técnico para fixação de identidade e qualidade do queijo Parmesão, Parmesano, Sbrinz, Reggiano E Reggianito. Brasilia, DF. Disponível em: <http:/ / extranet.agricultura.gov.br/sislegis-consulta/servlet/ VisualizarAnexo?id=2501>. Acesso em: 06 jun. 2017.

BRASIL. Ministério da Agricultura, Pecuária e Abastecimento. Portaria no 355 de 04 de setembro de 1997. Regulamento técnico para fixação de identidade e qualidade de queijo em pó. Brasilia, DF. Disponível em: <https:/ / wp.ufpel.edu.br/inspleite/ files/2016/03/Portaria-n\%C2\%B0-355-de-4-de-setembro-de-1997.pdf>.

BRASIL. Ministério da Agricultura, Pecuária e Abastecimento. Portaria no 357 de 04 de setembro de 1997. Regulamento técnico para fixação de identidade e qualidade do queijo ralado. Brasilia, DF. Disponível em: <http:/ / www.cidasc.sc.gov.br/ inspecao/files/2012/08/ PORTARIA-MA-357-DE-04_09_1997.pdf>. Acesso em: 06 jun. 2017.

BRASIL. Ministério da Agricultura, Pecuária e Abastecimento. Portaria no 358 de 04 de setembro de 1997. Regulamento técnico para fixação de identidade e qualidade do queijo Prato. Brasilia, DF. Disponível em: <http:/ / extranet.agricultura.gov.br/ sislegis-consulta/servlet/ VisualizarAnexo?id=2468>. Acesso em: 06 jun. 2017.

BRASIL. Ministério da Agricultura, Pecuária e Abastecimento. Portaria no 359 de 04 de setembro de 1997. Regulamento técnico para fixação de identidade e qualidade de Requeijão ou Requesón. Brasilia, DF. Disponível em: <http:/ / extranet.agricultura. gov.br/sislegis- consulta/servlet/VisualizarAnexo?id=2467>. Acesso em: 06 jun. 2017.

BRASIL. Ministério da Agricultura, Pecuária e Abastecimento. Portaria no 360 de 04 de setembro de 1997. Regulamento técnico para fixação de identidade e qualidade do queijo Danbo. Brasilia, DF. Disponível em: <http:/ / www.crmvgo.org.br/ legislacao/leite/ POR00000360.pdf>. Acesso em: 06 jun. 2017.

BRASIL. Ministério da Agricultura, Pecuária e Abastecimento. Portaria no 361 de 04 de setembro de 1997. Regulamento técnico para fixação de identidade e qualidade do queijo Tilsit. Brasilia, DF. Disponível em: <http://www.cidasc.sc.gov.br/ inspecao/files /2012/08/ Portaria-n\%C2\%BA-361-de-04-de-setembro-de-1997.pdf>. Acesso em: 06 jun. 2017.

BRASIL. Ministério da Agricultura, Pecuária e Abastecimento. Portaria no 362 de 04 de setembro de 1997. Regulamento técnico para fixação de identidade e qualidade de queijo Tybo. Brasilia, DF. Disponível em: < https://wp.ufpel.edu.br/inspleite/ files /2016/03/Portaria-n\%C2\%B0-362-de-4-de-setembro-de-1997.pdf>. Acesso em: 06 jun. 2017.

BRASIL. Ministério da Agricultura, Pecuária e Abastecimento. Portaria n 363 de 04 de setembro de 1997. Regulamento técnico para fixação de identidade e qualidade de queijo Pategrás Sandwich. Brasilia, DF. Disponível em: < https://wp.ufpel.edu. 
br/inspleite/files /2016/03/Portaria-n\%C2\%B0-363-de-4-de-setembro-de-1997.pdf>. Acesso em: 06 jun. 2017.

BRASIL. Ministério da Agricultura, Pecuária e Abastecimento. Portaria no 364 de 04 de setembro de 1997. Regulamento técnico para fixação de identidade e qualidade do queijo Mozzarella (Muzzarella ou Mussarela). Brasilia, DF. Disponível em: <http:/ / extranet. agricultura.gov.br/sislegis-consulta/servlet/ VisualizarAnexo?id=2462>. Acesso em: 06 jun. 2017.

BRASIL. Ministério da Agricultura, Pecuária e Abastecimento. Portaria no 365 de 04 de setembro de 1997. Regulamento técnico para fixação de identidade e qualidade de queijo Tandil. Brasilia, DF. Disponível em: < https://wp.ufpel.edu.br/inspleite/ files/2016/03/Portaria-n\%C2\%B0-365-de-4-de-setembro-de-1997.pdf >. Acesso em: 06 jun. 2017.

EVANGELISTA-BARRETO, Norma S.; SANTOS, Gleyde C. da F.; SOUZA, Jeferson dos S.; BERNARDES, Fernanda de S.; SILVA, Irana P. Queijos artesanais como veículo de contaminação de Escherichia coli e estafilococos coagulase positiva resistentes a antimicrobianos. Revista Brasileira de Higiene e Sanidade Animal, v.10, n.1, p. 55 - 67, jan./mar. 2016. ISSN: 1981-2965. Disponível em: < http://www. higieneanimal.ufc.br/seer/index.php/ higieneanimal/article/view/301/1457>. Acesso em: 6 dez. 2017.

FORSYTHE, Stephen J. Microbiologia da Segurança Alimentar. Tradução de Maria Carolina Minardi Guimarães e Cristina Leonhardt. Porto Alegre: Artmed, 2013.

GALINARI, Éder; NÓBREGA, Juliana E. da; ANDRADE, Nélio J. de; FERREIRA, Célia L. L. F. Microbiological aspects of the biofilm on wooden utensils used to make a Brazilian artisanal cheese. Brazilian Journal of Microbiology. v.45, n.2, p. $713-720$, 2014. ISSN: 1678-4405. Disponível em: < http:/ / www.scielo.br/scielo.php?script=sci_ arttext\&pid=S1517-83822014000200047> $>$. Acesso em: 4 abr. 2017.

MADIGAN, Michael T.; MARTINKO, John M.; PARKER, Jack. Microbiologia de Brock. Tradução e revisão técnica de Cynthia Maria Kyaw. $10^{a}$ ed. São Paulo: Prendice Hall, 2004.

MARINHEIRO, Mariana F. et al. Qualidade microbiológica de queijo mussarela em peça e fatiado. Semina: Ciências Agrárias, Londrina, v.36, p.1329-1334, mayo/jun. 2015, doi: 10.5433/1679-0359.2015v36n3p1329. Disponível em: < http:/ / www.redalyc. org $/$ articulo.oa?id= 445744148043 >. Acesso em: 6 dez. 2017.

MELLO, Eliane Z. de; ARMACHUK, Maquéle A. Avaliação do queijo colonial durante a maturação: modificações físico-químicas e microbiológicas. Repositório de outras coleções abertas (ROCA), Francisco Beltrão, set. 2013. Disponível em: <http:/ / repositorio.roca.utfpr.edu.br/jspui/handle/1/2087>. Acesso em: 2 abr. 2018.

OLIVEIRA, Aline M.; KURIHARA, Ricardo Y.; SILVA, Francine F. da; SILVA, Fernando de G.; JUNIOR, José C. R.; BELOTI, Vanerli. Condições higiênico-sanitárias 
da produção de queijos tipo Mussarela e Minas Frescal comercializados no norte do Paraná. Revista Institucional Laticínios Cândido Tostes, Juiz de Fora, v. 72, n. 1, p. 4047, jan./mar. 2017, doi: 10.14295/2238-6416.v72i1.556. Disponível em: < https:/ /www. revistadoilct.com.br/rilct/article/view/556/425>. Acesso em: 6 dez. 2017.

RAIMANN, Viviane I. Avaliação microbiológica de queijos mussarela produzidos na região sudoeste do Paraná. Repositório digital institucional da UFPR (DSPACE), Curitiba, ago. 2011. Disponível em: < https:/ / acervodigital. ufpr.br/ handle/1884/38611>. Acesso em: 3 abr. 2018.

REZENDE, Patrick H. L.; MENDONÇA, Eliane P.; MELO, Roberta T. de; COELHO, Letícia R.; MONTEIRO, Guilherme P.; ROSSI, Daise A. Aspectos sanitários do queijo Minas artesanal comercializado em feiras livres. Revista Institucional Laticínios Cândido Tostes, Juiz de Fora, n. 377, 65: 36-42, nov./dez. 2010. Disponível em: < https:/ / www.revistadoilct.com.br/rilct/article/ view/148>. Acesso em: 15 dez. 2017.

SILVA, Francisco R. da et al. Conservação e controle de qualidade de queijos:

Revisão. Pubvet, Maringá, v.11, n.4, p.333-341, abr. 2017. ISSN 1982.1263. Disponível em: <http:/ / www.pubvet.com.br/artigo/3752/conservaccedilatildeo-e-controle-dequalidade-de-queijos-revisatildeo>. Acesso em: 6 dez. 2017.

SILVA, Sirlene A. de; FURTADO, Sérvulo C.; VARGAS, Bianca L. Avaliação microbiológica do queijo Coalho produzido com leite pasteurizado sob refrigeração. Revista Nanbiquara 2017, vol 6, v1, jan./jun. 2016. ISSN 2179-5053. Disponível em: <http:/ / periodicos.fametro.edu.br/index.php/nanbiquara/article/ view/245>. Acesso em: 13 dez. 2017.

TONDO, Eduardo C.; BARTZ, Sabrina. Microbiologia e Sistema de Gestão de Segurança de Alimentos. Porto Alegre: Sulina, 2011.

TORTORA, Gerard J.; FUNKE, Berdell R.; CASE Christine L. Microbiologia. Tradução Aristóbolo Mendes da Silva et al. 10ª ed. Porto Alegre: Artmed, 2012. 